\title{
Measurement-induced spatial modulation of spontaneous decay and photon arrival times
}

\author{
Joachim von Zanthier, ${ }^{1}$ Thierry Bastin, ${ }^{2}$ and Girish S. Agarwal ${ }^{3}$ \\ ${ }^{1}$ Institut für Optik, Information und Photonik, Universität Erlangen-Nürnberg, 91058 Erlangen, Germany \\ ${ }^{2}$ Institut de Physique Nucléaire, Atomique et de Spectroscopie, Université de Liège au Sart Tilman, 4000 Liège, Belgium \\ ${ }^{3}$ Department of Physics, Oklahoma State University, Stillwater, Oklahoma 74078-3072, USA \\ (Received 28 March 2006; published 19 December 2006; publisher error corrected 20 December 2006)
}

\begin{abstract}
We report a way of manipulating the spontaneous emission process leading to a spatial modulation of spontaneous decay. The effect is observed in the case of coherently driven atoms separated by less than a transition wavelength. It is quantified by Glauber's photon-photon second-order correlation function. We show that the photon arrival time, usually regarded as an entirely random process, depends not only on where a photon is detected but also on where a former photon had been recorded previously. Our results shed light on the unexpected consequences of state reduction and entanglement for the fundamental process of spontaneous emission.
\end{abstract}

DOI: 10.1103/PhysRevA.74.061802

PACS number(s): 42.50.Dv, 03.65.Ud, 42.50.Ar, 42.50.Fx

Spontaneous deexcitation of atomic systems into their ground state is a basic fact of nature and has been known since the early days of quantum mechanics [1]. It results from the dynamical interaction between an excited atom and the electromagnetic fluctuations of the vacuum. It is also well known that this fundamental process can be modified in various ways by (i) changing the properties of the vacuum, e.g., using a cavity or metallic or dielectric interfaces [2-8], (ii) employing external laser fields $[9,10]$, (iii) continuously monitoring the decay process [11], or (iv) placing the system in a cooperative environment [12], leading to the collective phenomena of subradiance and superradiance [13-16]. Here we report a further aspect of manipulating the spontaneous emission process: namely, a spatial modulation of spontaneous decay in the case of coherently driven correlated atoms. The effect is quantified by calculating the photon-photon second-order correlation function, which describes the probability of detecting a photon at $\mathbf{r}_{2}$ and time $t+\tau$ after a photon at $\mathbf{r}_{1}$ and time $t$ has been recorded. The phenomenon is found to result from two kinds of correlations generated between the atoms, one from the dipole-dipole interaction when the particles are sufficiently close to each other [12] and the other by a back action on the atomic system when a photon emitted by the atoms is detected and the photonic detection does not distinguish between the atoms [17-20]. The spatial dependence of the spontaneous decay rate is distinct from standard position-dependent retardation phenomena. It is also not to be confounded with the usual intensity pattern of a single or several atoms which merely reflects the spatial fluorescence distribution of the corresponding multipolar atomic transitions and their possible interferences. In the latter case, for a single or uncorrelated atoms, the temporal behavior of the photon arrival time is the same in all directions.

To understand the outlined behavior, we consider two identical atoms 1 and 2 at fixed positions $\mathbf{x}_{1}$ and $\mathbf{x}_{2}$ with internal levels $|e\rangle$ and $|g\rangle$, dipolar transition frequency $\omega$ $=2 \pi c / \lambda$, and single-atom free-space Einstein $A$ coefficient $2 \gamma$. This system is conveniently described in the Dicke basis [12] $\left\{|g\rangle:=|g, g\rangle,|e\rangle:=|e, e\rangle,|s\rangle:=\frac{1}{\sqrt{2}}(|e, g\rangle+|g, e\rangle),|a\rangle\right.$ $\left.:=\frac{1}{\sqrt{2}}(|e, g\rangle-|g, e\rangle)\right\}$, which forms a set of eigenstates of the combined system. From the work of Dicke [12] we know that correlations between the two atoms are produced if the interatomic spacing $x_{12} \equiv\left|\mathbf{x}_{1}-\mathbf{x}_{2}\right|$ is reduced down to or below the transition wavelength $\lambda$. Correlations between the atomic dipoles are generated because the emission or absorption of a photon cannot be assigned to a specific particle any longer so that the atom-field interaction has to be symmetric with respect to atomic permutation. As a consequence, an imbalance appears among the possible transitions between the Dicke states that causes the atomic system to decay with different rates, one enhanced and the other reduced with respect to the free-atom spontaneous emission rate $2 \gamma[12-16]$. The decay rates towards and from the symmetric state $|s\rangle$ [antisymmetric state $|a\rangle]$ are given by $2 \gamma^{+}=2(\gamma+\Delta \gamma)\left[2 \gamma^{-}\right.$ $=2(\gamma-\Delta \gamma)]$, where $\Delta \gamma$ is the imaginary part of

$$
\begin{aligned}
\Delta \Omega+i \Delta \gamma= & \frac{3}{2} \gamma e^{-i k x_{12}}\left[\frac{\cos ^{2} \theta-1}{k x_{12}}\right. \\
& \left.+\left(1-3 \cos ^{2} \theta\right)\left(\frac{i}{\left(k x_{12}\right)^{2}}+\frac{1}{\left(k x_{12}\right)^{3}}\right)\right] .
\end{aligned}
$$

Here, $\pm \Delta \Omega$ represents the level shift of the states $|s\rangle$ and $|a\rangle$, respectively, due to the dipole-dipole interaction between the two atoms, $k=\omega / c$, and $\theta$ is the angle between the atomic dipole moment $\mathbf{d}$ and $\mathbf{x}_{12}$. The Dicke states, level shifts, and modified transition rates are schematically shown in Fig. 1.

Next, we investigate the two time photon-photon secondorder correlation function [21]

$$
g^{(2)}\left(\mathbf{r}_{1}, t ; \mathbf{r}_{2}, t+\tau\right)=\frac{G^{(2)}\left(\mathbf{r}_{1}, t ; \mathbf{r}_{2}, t+\tau\right)}{G^{(1)}\left(\mathbf{r}_{1}, t\right) G^{(1)}\left(\mathbf{r}_{2}, t\right)},
$$

where $G^{(1)}(\mathbf{r}, t)$ and $G^{(2)}\left(\mathbf{r}_{1}, t ; \mathbf{r}_{2}, t+\tau\right)$ are given by

$$
G^{(1)}(\mathbf{r}, t)=2 \gamma u(\mathbf{r}) \sum_{\mu, \nu=1}^{2} e^{\left[i k \mathbf{x}_{\mu, \nu} \hat{\mathbf{r}}\right]}\left\langle\sigma_{\mu}^{+}(t) \sigma_{\nu}^{-}(t)\right\rangle
$$




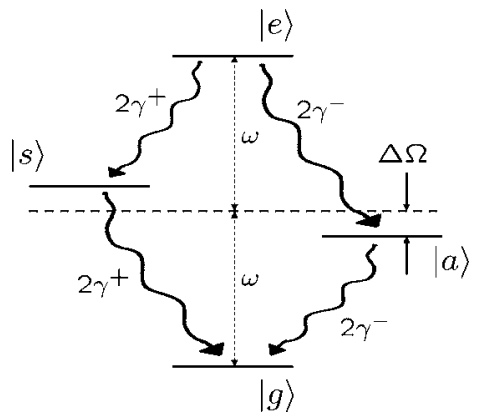

FIG. 1. Dicke states $|g\rangle,|e\rangle,|s\rangle$, and $|a\rangle$ of two identical twolevel atoms with corresponding level shifts $\pm \Delta \Omega$ and transition rates $2 \gamma^{ \pm}$.

$$
\begin{aligned}
G^{(2)}\left(\mathbf{r}_{1}, t ; \mathbf{r}_{2}, t+\tau\right)= & 4 \gamma^{2} u\left(\mathbf{r}_{1}\right) u\left(\mathbf{r}_{2}\right) \sum_{\mu, \nu, \lambda, \rho=1}^{2} e^{\left[i k\left(\mathbf{x}_{\lambda, \nu} \hat{\mathbf{r}}_{1}+\mathbf{x}_{\rho, \mu}, \hat{\mathbf{r}}_{2}\right)\right]} \\
& \times\left\langle\sigma_{\lambda}^{+}(t) \sigma_{\rho}^{+}(t+\tau) \sigma_{\mu}^{-}(t+\tau) \sigma_{\nu}^{-}(t)\right\rangle
\end{aligned}
$$

Here $\hat{\mathbf{r}}=\mathbf{r} /|\mathbf{r}|, \sigma_{\mu}^{-}=|g\rangle_{\mu \mu}\langle e|$ is the atomic lowering operator for atom $\mu(\mu=1,2), \sigma_{\mu}^{+}=\left(\sigma_{\mu}^{-}\right)^{\dagger}, \mathbf{x}_{\mu, \nu}=\mathbf{x}_{\mu}-\mathbf{x}_{\nu}$, and $u(\mathbf{r})$ $=\left(\omega^{4} d^{2} / 2 r^{2} c^{4} \pi \epsilon_{0}\right) \sin ^{2} \varphi$ describes the usual dipole intensity pattern, with $\varphi$ the angle between the observation direction $\mathbf{r}$ and the dipole moment $\mathbf{d}$. Equation (2) can also be written in the form

$$
g^{(2)}\left(\mathbf{r}_{1}, t ; \mathbf{r}_{2}, t+\tau\right)=\frac{P\left(\mathbf{r}_{2}, t+\tau \mid \mathbf{r}_{1}, t\right)}{P\left(\mathbf{r}_{2}, t\right)},
$$

where $P(\mathbf{r}, t)$ is the probability of detecting a photon at position $\mathbf{r}$ and time $t$ and $P\left(\mathbf{r}_{2}, t+\tau \mid \mathbf{r}_{1}, t\right)$ is the conditional probability of finding a photon at $\mathbf{r}_{2}$ and $t+\tau$ assuming that a photon at $\mathbf{r}_{1}$ and $t$ has been recorded. In case of a single atom we know that the overall time behavior of $g^{(2)}\left(\mathbf{r}_{1}, t ; \mathbf{r}_{2}, t+\tau\right)$-i.e., the photon arrival time-is independent of the direction of observation and is governed by the free-atom spontaneous decay rate $2 \gamma$ modified by the Rabi frequency of the driving field $[22,23]$.

The probabilities $P\left(\mathbf{r}_{1}, t\right)$ and $P\left(\mathbf{r}_{2}, t+\tau \mid \mathbf{r}_{1}, t\right)$ can be calculated-using the quantum regression theorem [24] from the dynamical behavior of the density operator $\rho$ via

$$
\begin{gathered}
P(\mathbf{r}, t)=\left\langle D^{\dagger}(\mathbf{r}) D(\mathbf{r})\right\rangle_{\rho(t)}, \\
P\left(\mathbf{r}_{2}, t+\tau \mid \mathbf{r}_{1}, t\right)=\left\langle D^{\dagger}\left(\mathbf{r}_{2}\right) D\left(\mathbf{r}_{2}\right)\right\rangle_{\rho^{\prime}(t+\tau)_{\mathbf{r}_{1}, t},}
\end{gathered}
$$

where $\rho(t)$ is the density operator of the two-atom system at time $t, \rho^{\prime}(t+\tau)_{\mathbf{r}_{1}, t}$ is the density operator at time $t+\tau$ assuming a photon has been detected at point $\mathbf{r}_{1}$ and time $t$, and $D(\mathbf{r})$ is the photon detector operator [21]

$$
D(\mathbf{r})=\sigma_{1}^{-}+e^{i \delta(\mathbf{r})} \sigma_{2}^{-},
$$

with $\delta(\mathbf{r})=k \hat{\mathbf{r}} \cdot \mathbf{x}_{12}$. In the case that the atoms are coherently driven by a laser of Rabi frequency $2 \Omega$ and wave vector $\mathbf{k}_{L}$, resonant with the single-atom transition $\omega$ and chosen (though not necessary) to be perpendicular to $\mathbf{x}_{12}$, the time evolution of the density operator $\rho$ in the interaction picture obeys the master equation [14]

$$
\begin{aligned}
\dot{\rho}= & -i \Omega \sum_{\mu=1}^{2}\left[e^{i \mathbf{k}_{L} \cdot \mathbf{x}_{\mu}} \sigma_{\mu}^{+}+\text {H.c. }, \rho\right]-i \Delta \Omega \sum_{\mu \neq \nu}\left[\sigma_{\mu}^{+} \sigma_{\nu}^{-}, \rho\right] \\
& -i \sum_{\mu, \nu} \gamma_{\mu \nu}\left(\sigma_{\mu}^{+} \sigma_{\nu}^{-} \rho+\rho \sigma_{\mu}^{+} \sigma_{\nu}^{-}-2 \sigma_{\mu}^{-} \rho \sigma_{\nu}^{+}\right) .
\end{aligned}
$$

where $\gamma_{\mu \nu}=\gamma$ for $\mu=\nu$ and $\gamma_{\mu \nu}=\Delta \gamma$ for $\mu \neq \nu$.

In Fig. 2(a), $g^{(2)}\left(\mathbf{r}_{1}, 0 ; \mathbf{r}_{2}, \tau\right)$ is plotted for a single detector at $\mathbf{r}$ in the case of a large distance between the two atoms $\left(x_{12}=20 \lambda\right)$. Here, the dipole-dipole interaction is negligible so that the results of $[19,26]$ are recovered: a strong modulation of $g^{(2)}(\mathbf{r}, 0 ; \mathbf{r}, \tau=0)$ with respect to $\mathbf{r}$ is observed, changing from sub-Poissonian statistics and antibunching to super-Poissonian statistics and bunching. The decay and growing time scales of $g^{(2)}(\mathbf{r}, 0 ; \mathbf{r}, \tau)$ are equal in this case, in accordance with $\gamma^{+}=\gamma^{-}=\gamma$, which holds for large distances $x_{12}$ between the two atoms [see Eq. (1)].

For small distances $\left(x_{12} \leq \lambda\right)$, we observe for $\tau=0$ still the same spatial modulation of $g^{(2)}(\mathbf{r}, 0 ; \mathbf{r}, \tau)$. However, the decay and growing times are now different, reflecting the difference in the decay constants $\gamma^{+}$and $\gamma^{-}$when the dipoledipole interaction is significant. A clear imbalance in the photon arrival times for the different spatial directions is observed in this case [see Fig. 2(b)].

In Fig. 2(c), the second-order correlation function is plotted for two detectors located in opposite directions. It shows that for particular detector positions [such that $\delta(\mathbf{r})= \pm \pi / 2$ ], complete antibunching is observed [i.e., $g^{(2)}(\mathbf{r}, 0 ;-\mathbf{r}, 0)=0$ ], while the imbalance in the photon arrival times for different spatial directions is preserved as in Fig. 2(b).

To understand this highly nonclassical behavior we recall that in addition to the correlations generated between the atomic dipoles in the case of $x_{12} \lesssim \lambda$ further correlations are produced if a single photon emitted by the two-atom system is detected and the photonic detection does not distinguish between the two atoms [17-20]. These correlations are generated even in case of distant atoms-i.e., in the absence of any interatomic interaction [17-20]. The production of correlations can be understood by investigating the reduced state of the atomic system after the detection of the first photon. According to the von Neumann projection postulate the reduced state reads

$$
\rho\left(\mathbf{r}_{1}\right)=\frac{D\left(\mathbf{r}_{1}\right) \tilde{\rho} D^{\dagger}\left(\mathbf{r}_{1}\right)}{\left\langle D^{\dagger}\left(\mathbf{r}_{1}\right) D\left(\mathbf{r}_{1}\right)\right\rangle_{\widetilde{\rho}}},
$$

with $\tilde{\rho}$ the steady-state density matrix just before detection. From Eq. (10) the density operator evolves again according to Eq. (9).

For the two detectors positioned at $\mathbf{r}_{i}(i=1,2)$ such that $\delta\left(\mathbf{r}_{i}\right)=2 n \pi\left[\delta\left(\mathbf{r}_{i}\right)=(2 n+1) \pi, n\right.$ integer $]$, Eq. (8) leads to

$$
D\left(\mathbf{r}_{i}\right)=\sigma_{1}^{-} \pm \sigma_{2}^{-} .
$$

In the first case, the detector operators $D\left(\mathbf{r}_{i}\right)$ are symmetric, which means that the atomic system is projected such that only photons decaying along the "symmetric decay channel" $|e\rangle \rightarrow|s\rangle \rightarrow|g\rangle$ are produced. In the second case, the detector operators are antisymmetric so that only photons decaying along the "antisymmetric decay channel" $|e\rangle \rightarrow|a\rangle \rightarrow|g\rangle$ are 

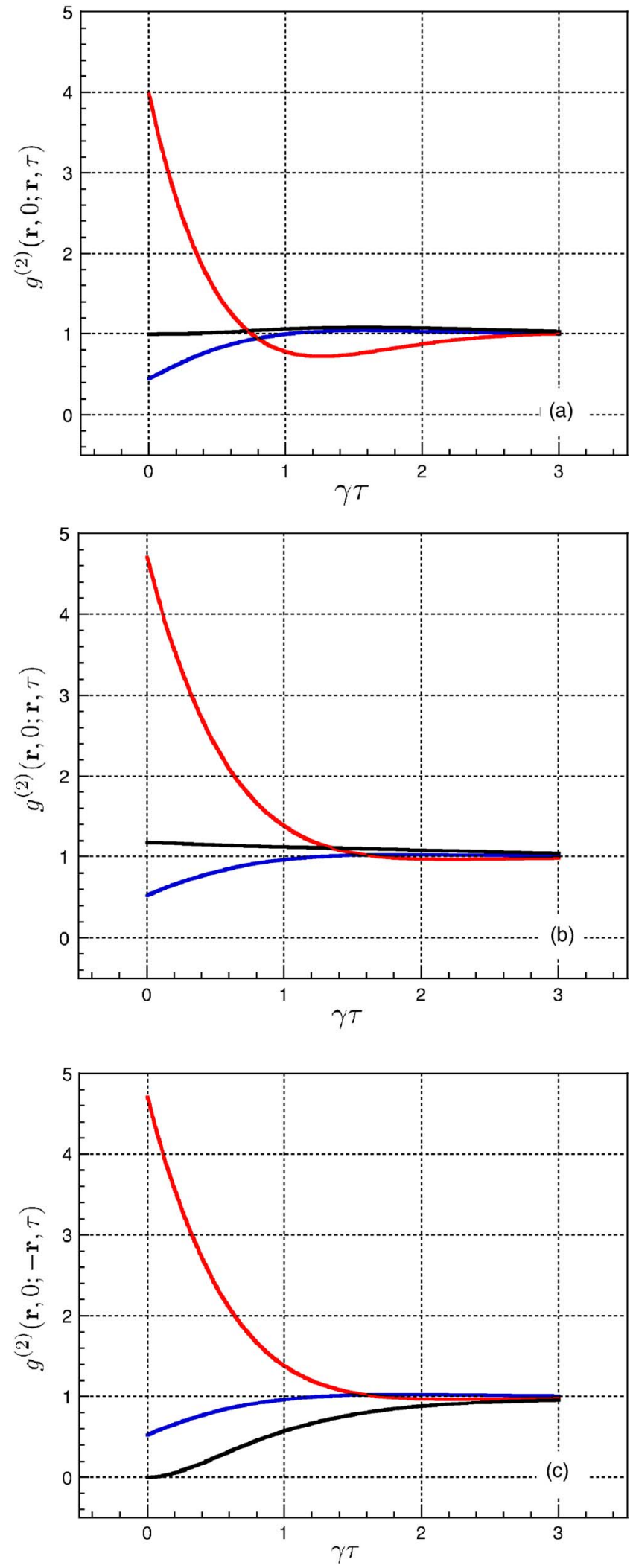

FIG. 2. (Color online) Correlation function $g^{(2)}\left(\mathbf{r}_{1}, 0 ; \mathbf{r}_{2}, \tau\right)$ with respect to $\tau$ for two coherently driven atoms. Parameters are $\theta=0$, $\Omega=0.7 \gamma$, (a) $\mathbf{r}_{1}=\mathbf{r}=\mathbf{r}_{2}, x_{12}=20 \lambda$, (b) $\mathbf{r}_{1}=\mathbf{r}=\mathbf{r}_{2}, x_{12}=\lambda / 2$, and (c) $\mathbf{r}_{1}=\mathbf{r}=-\mathbf{r}_{2}, x_{12}=\lambda / 2$. In (a) and (b), the curves from bottom to top refer to $\delta(\mathbf{r})=0, \pm \pi / 2, \pm \pi$. In (c), the curves from bottom to top refer to $\delta(\mathbf{r})= \pm \pi / 2,0, \pm \pi$. generated (see Fig. 1). Combining both ways of generating correlations between the two atoms one notices that depending on the detector positions one can select not only the channel along which the two atom system will decay but also the rate at which the photons are emitted (see Fig. 1). Different photon arrival times are thus observable in different directions, depending on where the spontaneously emitted photons are recorded.

For the two detectors located at $\mathbf{r}_{1}=\mathbf{r}=-\mathbf{r}_{2}$ such that $\delta(\mathbf{r})=(2 n+1) \pi / 2, n$ even (odd), we get, from Eq. (8), $D(\mathbf{r})$ $=\sigma_{1}^{-} \pm i \sigma_{2}^{-}$and $D(-\mathbf{r})=\sigma_{1}^{-} \mp i \sigma_{2}^{-}$. In both configurations, $D(\mathbf{r}) D(-\mathbf{r})=0$, so that two subsequent detections can never occur and perfect antibunching is observed.

These behaviors can be further elucidated by considering a pulsed excitation where the two atoms are initially in the double-excited state $|e\rangle$. For the two detectors located at $\mathbf{r}_{1}$ and $\mathbf{r}_{2}$ we obtain in this case, from Eqs. (5)-(9) for the second-order correlation function,

$$
\begin{aligned}
g^{(2)}\left(\mathbf{r}_{1}, 0 ; \mathbf{r}_{2}, \tau\right)= & e^{-2 \gamma \tau}\left(e^{-2 \Delta \gamma \tau} \cos ^{2} \frac{\delta\left(\mathbf{r}_{1}\right)}{2} \cos ^{2} \frac{\delta\left(\mathbf{r}_{2}\right)}{2}\right. \\
& +e^{2 \Delta \gamma \tau} \sin ^{2} \frac{\delta\left(\mathbf{r}_{1}\right)}{2} \sin ^{2} \frac{\delta\left(\mathbf{r}_{2}\right)}{2} \\
& \left.+\sin \delta\left(\mathbf{r}_{1}\right) \sin \delta\left(\mathbf{r}_{2}\right) \frac{\cos (2 \Delta \Omega \tau)}{2}\right) .
\end{aligned}
$$

Equation (12) is $2 \pi$ periodic in $\delta\left(\mathbf{r}_{1}\right)$ and $\delta\left(\mathbf{r}_{2}\right)$. The full range of effects is thus observed when these parameters vary between $-\pi$ and $\pi$. This can be achieved whenever the interatomic distance $x_{12} \geqslant \lambda / 2$. For smaller distances the range of variation $\pm k x_{12}$ of $\delta(\mathbf{r})$ is reduced to values $< \pm \pi$.

In case that a single detector at $\mathbf{r}=\mathbf{r}_{1}=\mathbf{r}_{2}$ is employed and for an interatomic spacing $x_{12}$ large compared to $\lambda$ (i.e., $\Delta \gamma \simeq 0 \simeq \Delta \Omega$ ), Eq. (12) simplifies to

$$
g^{(2)}(\mathbf{r}, 0 ; \mathbf{r}, \tau)=e^{-2 \gamma \tau}
$$

i.e., a mean photon arrival time $\sim(2 \gamma)^{-1}$ is observed, independent from the direction of observation, as expected. However, for $x_{12} \lesssim \lambda$ (i.e., $\Delta \gamma \neq 0 \neq \Delta \Omega$ ), we see from Eq. (12) that the photon arrival time is modulated as a function of the detector position r. In particular, for the detector located such that $\delta(\mathbf{r})=0$, Eq. (12) simplifies to

$$
g^{(2)}(\mathbf{r}, 0 ; \mathbf{r}, \tau)=e^{-2 \gamma^{+} \tau},
$$

whereas for $\delta(\mathbf{r})= \pm \pi$ we obtain

$$
g^{(2)}(\mathbf{r}, 0 ; \mathbf{r}, \tau)=e^{-2 \gamma^{-} \tau} .
$$

Note that for $\delta(\mathbf{r})=0[\delta(\mathbf{r})= \pm \pi]$ the detection of the first photon transforms the system from the nonentangled state $|e\rangle$ to the entangled state $|s\rangle(|a\rangle)$. As the two states $|s\rangle$ and $|a\rangle$ have not the same spontaneous decay rate $\left(2 \gamma^{+}\right.$and $2 \gamma^{-}$, respectively), different emission rates are monitored in different spatial directions. Moreover, since the atoms are entangled after the detection of the first photon, the dependence of the arrival time of the second photon on the detector location becomes a nonlocal phenomenon. 


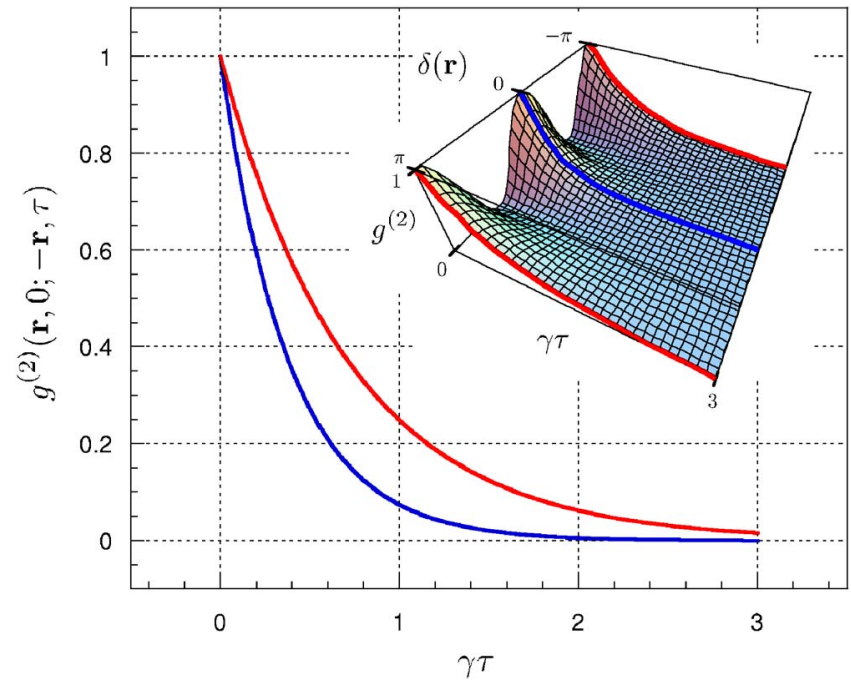

FIG. 3. (Color online) Correlation function $g^{(2)}(\mathbf{r}, 0 ;-\mathbf{r}, \tau)$ with respect to $\tau$ for pulsed excitation [inset: with respect to $\tau$ and $\delta(\mathbf{r})$ ]. Parameters are $\theta=0$ and $x_{12}=\lambda / 2$. The curves from bottom to top refer to $\delta(\mathbf{r})=0, \pm \pi$.

This aspect becomes more apparent if the two detectors are located at different positions $\mathbf{r}_{1} \neq \mathbf{r}_{2}$ : As can be seen from Eq. (12) the detection of a photon at $\mathbf{r}_{1}$ influences the arrival time of the second photon at $\mathbf{r}_{2}$. The photon arrival time depends thus on where the first photon was detected and where the second photon is to be detected. This is yet another example of the nonlocal character of the measurement process in the case that entangled states are considered [25]; however, in this case it applies to the fundamental phenomenon of spontaneous emission, usually regarded as an entirely random process.

In particular, for the two detectors located at $\mathbf{r}_{1}=\mathbf{r}=-\mathbf{r}_{2}$, the overall behavior of $g^{(2)}(\mathbf{r}, 0 ;-\mathbf{r}, \tau)$ as a function of $\delta(\mathbf{r})$ is shown in Fig. 3. This figure exhibits clearly the spatial modulation of the arrival time of the second photon [Eqs. (14) and (15) still hold for the two detectors located in opposite directions], as well as the occurrence of perfect antibunching for $\delta(\mathbf{r})= \pm \pi / 2$.

Note that our results can be used to demonstrate experimentally in a simple manner the subradiance and superradiance behavior of a two-atom system simultaneously. For that purpose the atoms need to be placed sufficiently close to each other so that the decay rates of the two decay channels are significantly modified. This has been experimentally realized for two trapped ions [27] and could be envisaged-in view of recent advances [28] -also for neutral atoms. The system is then automatically forced to subradiate or superradiate, depending solely on where the photons are recorded.

We gratefully acknowledge financial support by the Dr. Hertha und Helmut Schmauser foundation.
[1] V. Weisskopf and E. Wigner, Z. Phys. 63, 54 (1930).

[2] E. M. Purcell, Phys. Rev. 69, 681 (1946).

[3] D. Kleppner, Phys. Rev. Lett. 47, 233 (1981).

[4] S. Haroche, Fundamental Systems in Quantum Optics (NorthHolland, Amsterdam, 1992).

[5] Cavity Quantum Electrodynamics, edited by P. R. Berman (Academic Press, Boston, 1994).

[6] K. H. Drexhage, in Progress in Optics, edited by E. Wolf (North-Holland, New York, 1974), Vol. 12.

[7] G. S. Agarwal, Phys. Rev. A 12, 1475 (1975).

[8] J. Eschner, Ch. Raab, F. Schmidt-Kaler, and R. Blatt, Nature (London) 413, 495 (2001).

[9] S.-Y. Zhu and M. O. Scully, Phys. Rev. Lett. 76, 388 (1996); H. R. Xia, C. Y. Ye, and S.-Y. Zhu, ibid. 77, 1032 (1996).

[10] E. Paspalakis and P. L. Knight, Phys. Rev. Lett. 81, 293 (1998).

[11] A. G. Kofman and G. Kurizki, Nature (London) 405, 546 (2000).

[12] R. H. Dicke, Phys. Rev. 93, 99 (1954).

[13] R. H. Lehmberg, Phys. Rev. A 2, 883 (1970).

[14] G. S. Agarwal, Quantum Statistical Theories of Spontaneous Emission and Their Relation to Other Approaches, Springer Tracts in Modern Physics, Vol. 70 (Springer, Berlin, 1974).

[15] M. Gross and S. Haroche, Phys. Rep. 93, 301 (1982).

[16] Z. Ficek and R. Tanaś, Phys. Rep. 372, 369 (2002).
[17] C. Cabrillo, J. I. Cirac, P. Garcia-Fernandez, and P. Zoller, Phys. Rev. A 59, 1025 (1999).

[18] L.-M. Duan, M. D. Lukin, J. I. Cirac, and P. Zoller, Nature (London) 414, 413 (2001).

[19] C. Skornia, J. von Zanthier, G. S. Agarwal, E. Werner, and H. Walther, Phys. Rev. A 64, 063801 (2001). For a recent experiment on the subject, see J. Beugnon et al., Nature (London) 440, 779 (2006).

[20] C. W. Chou, H. de Riedmatten, D. Felinto, S. V. Polyakov, S. J. van Enk, and H. J. Kimble, Nature (London) 438, 828 (2005).

[21] R. J. Glauber, Phys. Rev. 130, 2529 (1963). We have chosen here a normalization that is different from that of Glauber so that we can deal with both transient and steady-state situations.

[22] H. J. Carmichael and D. F. Walls, J. Phys. B 9, 1199 (1976).

[23] F. Diedrich and H. Walther, Phys. Rev. Lett. 58, 203 (1987).

[24] M. Lax, Phys. Rev. 172, 350 (1968).

[25] Quantum Theory and Measurement, edited by J. A. Wheeler and W. H. Zurek (Princeton University Press, Princeton, 1983).

[26] C. Schön and A. Beige, Phys. Rev. A 64, 023806 (2001).

[27] R. G. DeVoe and R. G. Brewer, Phys. Rev. Lett. 76, 2049 (1996).

[28] I. Dotsenko, W. Alt, M. Khudaverdyan, S. Kuhr, D. Meschede, Y. Miroshnychenko, D. Schrader, and A. Rauschenbeutel, Phys. Rev. Lett. 95, 033002 (2005). 